(2) Open Access Full Text Article

CORRIGENDUM

\title{
Adapting Inhaled Medication Practice in COPD and Asthma to Avoid Funding the Tobacco Industry [Corrigendum]
}

Capstick TGD, Hopkinson NS. Int $J$ Chron Obstruct

Pulmon Dis. 2021;16:2917-2923.
The authors apologize for these errors and advise they do not affect the results of the paper.

The authors have advised there are minor errors in Table 2

on pages 2920-2921. The correct Table 2 is as follows.

Table 2 Potential Alternatives to Inhalers for COPD and Asthma That are Not Associated with Vectura

\begin{tabular}{|c|c|c|c|c|}
\hline $\begin{array}{l}\text { Brand } \\
\text { Name }\end{array}$ & Generic Drugs & Licence & $\begin{array}{l}\text { Manufacturerl } \\
\text { Partners }\end{array}$ & Alternative Options \\
\hline $\begin{array}{l}\text { Flutiform } \\
\text { pMDI }\end{array}$ & $\begin{array}{l}\text { Fluticasone Propionate/ } \\
\text { Formoterol }\end{array}$ & Asthma & $\begin{array}{l}\text { Kyorin } \\
\text { MundiPharma }\end{array}$ & 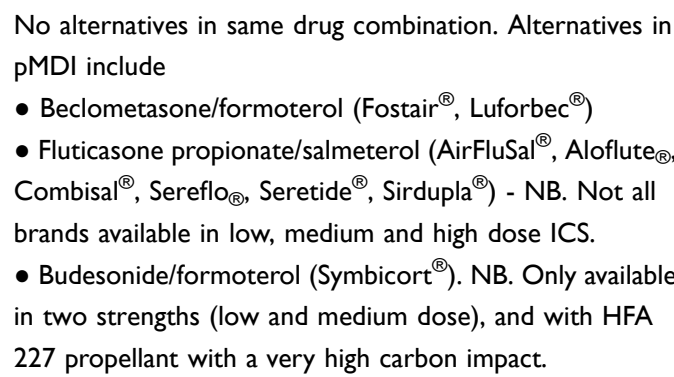 \\
\hline $\begin{array}{l}\text { Flutiform } \\
\text { K-haler }\end{array}$ & $\begin{array}{l}\text { Fluticasone Propionate/ } \\
\text { Formoterol }\end{array}$ & Asthma & MundiPharma & $\begin{array}{l}\text { Flutiform K-haler discontinued in UK April 202I. No ICS/ } \\
\text { LABA available in breath-actuated PMDI devices. } \\
\text { For alternatives in PMDI, see Flutiform PMDI, above. }\end{array}$ \\
\hline $\begin{array}{l}\text { Ultibro } \\
\text { Breezhaler }\end{array}$ & Indacaterol/glycopyrronium & COPD & Novartis & $\begin{array}{l}\text { DPI alternative: } \\
\text { - Duaklir Genuair (formoterol/aclidinium) } \\
\text { Aerosol alternatives: } \\
\text { - Bevespi Aerosphere pMDI (formoterol/glycopyrronium) } \\
\text { - Spiolto Respimat (olodaterol/tiotropium) }\end{array}$ \\
\hline $\begin{array}{l}\text { Seebri } \\
\text { Breezhaler }\end{array}$ & Glycopyrronium & COPD & Novartis & $\begin{array}{l}\text { DPI alternative: } \\
\text { - Eklira Genuair (aclidinium) } \\
\text { - Tiotropium (Spiriva }{ }^{\circledR} \text { HandiHaler, Braltus }{ }^{\circledR} \text { Zonda, } \\
\text { Tiogiva }{ }^{\circledR} \text { MRX003-R inhaler, Acopair }{ }^{\circledR} \text { NeumoHaler) } \\
\text { Aerosol alternative: } \\
\text { - Spiriva Respimat (tiotropium) }\end{array}$ \\
\hline
\end{tabular}

(Continued) 
Table 2 (Continued).

\begin{tabular}{|c|c|c|c|c|}
\hline $\begin{array}{l}\text { Brand } \\
\text { Name }\end{array}$ & Generic Drugs & Licence & $\begin{array}{l}\text { Manufacturer/ } \\
\text { Partners }\end{array}$ & Alternative Options \\
\hline $\begin{array}{l}\text { Enerzair } \\
\text { Breezhaler }\end{array}$ & $\begin{array}{l}\text { Mometasone/Indacaterol/ } \\
\text { glycopyrronium }\end{array}$ & Asthma & Novartis & $\begin{array}{l}\text { There are no alternative ICS/LABA/LAMA DPI inhalers } \\
\text { licensed for use in asthma. DPI alternatives would require } \\
\text { use of a suitable ICS/LABA DPI in combination with Spiriva } \\
\text { Respimat (the only licensed LAMA for asthma). } \\
\text { Aerosol alternative: } \\
\text { - Trimbow MDI (beclometasone/formoterol/ } \\
\text { glycopyrronium). NB. Trimbow is licensed in asthma as } \\
\text { a medium dose ICS, whilst Enerzair is a high dose ICS, so } \\
\text { a switch would represent an ICS dose reduction. }\end{array}$ \\
\hline $\begin{array}{l}\text { AirFluSal } \\
\text { Forspiro }\end{array}$ & $\begin{array}{l}\text { Fluticasone Propionate/ } \\
\text { Salmeterol }\end{array}$ & $\begin{array}{l}\text { Asthma, } \\
\text { COPD }\end{array}$ & Sandoz & $\begin{array}{l}\text { Alternative DPIs include: } \\
\text { - Fluticasone propionate/salmeterol DPIs: Seretide } \\
\text { Accuhaler, Fixkoh }^{\circledR} \text { Airmaster, Fusacomb }{ }_{\circledast} \text { Easyhaler, } \\
\text { Stalpex }{ }^{\circledR} \text { G7 inhaler } \\
\text { Alternative DPls for other ICS/LABA drugs include: } \\
\text { - Budesonide/formoterol: DuoResp }{ }^{\circledR} \text { Spiromax, Fobumix }{ }^{\circledR} \\
\text { Easyhaler, Symbicort }{ }^{\circledR} \text { Turbohaler } \\
\text { - Beclometasone/formoterol: (Fostair }{ }^{\circledR} \text { NEXThaler) }\end{array}$ \\
\hline $\begin{array}{l}\text { AirBuFo } \\
\text { Forspiro }^{\text {a }}\end{array}$ & Budesonide/formoterol & $\begin{array}{l}\text { Asthma, } \\
\text { COPD }\end{array}$ & Sandoz & $\begin{array}{l}\text { Not licensed in the UK. } \\
\text { Alternative DPIs include: } \\
\text { - Budesonide/formoterol: DuoResp }{ }^{\circledR} \text { Spiromax, Fobumix } \\
\text { Easyhaler, Symbicort }{ }^{\circledR} \text { Turbohaler } \\
\text { Alternative DPIs for other ICS/LABA drugs include: } \\
\text { - Beclometasone/formoterol: (Fostair }{ }^{\circledR} \text { NEXThaler) } \\
\text { - Fluticasone propionate/Salmeterol DPls: Seretide }{ }^{\circledR} \\
\text { Accuhaler, Fixkoh }^{\circledR} \text { Airmaster, Fusacomb }{ }^{\circledR} \text { Easyhaler, } \\
\text { Stalpex }^{\circledR} \text { G7 inhaler }\end{array}$ \\
\hline $\begin{array}{l}\text { Fluticasone } \\
\text { Propionate } \\
\text { and } \\
\text { Salmeterol }\end{array}$ & $\begin{array}{l}\text { Fluticasone Propionate/ } \\
\text { Salmeterol }\end{array}$ & $\begin{array}{l}\text { Asthma, } \\
\text { COPD }\end{array}$ & Hikma & $\begin{array}{l}\text { Not licensed in the UK. } \\
\text { Alternative DPIs include: } \\
\text { Fluticasone Propionate/Salmeterol DPIs: Seretide }{ }^{\circledR} \\
\text { Accuhaler, Fixkoh }{ }^{\circledR} \text { Airmaster, Fusacomb }{ }^{\circledR} \text { Easyhaler, } \\
\text { Stalpex }{ }^{\circledR} \text { G7 inhaler }\end{array}$ \\
\hline Breelib $^{c}$ & $\begin{array}{l}\text { Nebuliser for adminstration of } \\
\text { Ventavis (iloprost), or generic } \\
\text { lloprost Zentiva }\end{array}$ & $\begin{array}{l}\text { Pulmonary } \\
\text { Hypertension }\end{array}$ & Bayer & $\begin{array}{l}\text { No other licensed iloprost nebuliser solution preparations } \\
\text { available }\end{array}$ \\
\hline Incruse Ellipta & Umeclidinium & COPD & GSK & $\begin{array}{l}\text { DPI alternative: } \\
\text { - Eklira Genuair (aclidinium) } \\
\text { - Tiotropium (Spiriva }{ }^{\circledR} \text { HandiHaler, Braltus }{ }^{\circledR} \text { Zonda, } \\
\text { Tiogiva }{ }^{\circledR} \text { MRX003-R inhaler, Acopair }{ }^{\circledR} \text { NeumoHaler) } \\
\text { Aerosol alternative: } \\
\text { - Spiriva Respimat (tiotropium) }\end{array}$ \\
\hline Anoro Ellipta & Vilanterol/Umeclidinium & COPD & GSK & $\begin{array}{l}\text { DPI alternative: } \\
\text { - Duaklir Genuair (formoterol/aclidinium) } \\
\text { Aerosol alternatives: } \\
\text { - Bevespi Aerosphere pMDI (formoterol/glycopyrronium) } \\
\text { - Spiolto Respimat (olodaterol/tiotropium) }\end{array}$ \\
\hline
\end{tabular}

(Continued) 
Table 2 (Continued).

\begin{tabular}{|c|c|c|c|c|}
\hline $\begin{array}{l}\text { Brand } \\
\text { Name }\end{array}$ & Generic Drugs & Licence & $\begin{array}{l}\text { Manufacturerl } \\
\text { Partners }\end{array}$ & Alternative Options \\
\hline $\begin{array}{l}\text { Relvar/Breo } \\
\text { Ellipta }\end{array}$ & Fluticasone furoate/vilanterol & $\begin{array}{l}\text { Asthma, } \\
\text { COPD }\end{array}$ & GSK & $\begin{array}{l}\text { No alternatives in same drug combination. DPI } \\
\text { alternatives: } \\
\text { - Fluticasone propionate/salmeterol DPIs: Seretide } \\
\text { Accuhaler, Fixkoh }{ }^{\circledR} \text { Airmaster, Fusacomb }{ }^{\circledR} \text { Easyhaler, } \\
\text { Stalpex }{ }^{\circledR} \text { G7 inhaler } \\
\text { - Budesonide/formoterol: DuoResp }{ }^{\circledR} \text { Spiromax, Fobumix }{ }^{\circledR} \\
\text { Easyhaler, Symbicort }{ }^{\circledR} \text { Turbohaler } \\
\text { - Beclometasone/formoterol: (Fostair }{ }^{\circledR} \text { NEXThaler) }\end{array}$ \\
\hline Trelegy Ellipta & $\begin{array}{l}\text { Fluticasone furoate/vilanterol/ } \\
\text { umeclidinium }\end{array}$ & COPD & GSK & $\begin{array}{l}\text { DPI alternative: } \\
\text { - Trimbow NEXThaler (beclometasone/formoterol/ } \\
\text { glycopyrronium). Available from September 202I. } \\
\text { Aerosol alternativies: } \\
\text { - Trimbow MDI (beclometasone/formoterol/ } \\
\text { glycopyrronium) } \\
\text { - Trixeo Aerosphere pMDI (budesonide/formoterol/ } \\
\text { glycopyrronium) }\end{array}$ \\
\hline
\end{tabular}

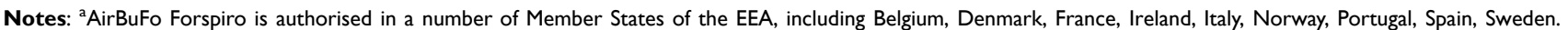
${ }^{b}$ Hikma Pharmaceuticals plc announced that they had resumed the launch of a generic version of Advair diskus in the US. ${ }^{c}$ Breelib is a nebuliser approved for the administration of iloprost (Ventavis ${ }^{\circledR}$ ) and generic lloprost Zentiva nebuliser solution to adult patients with primary pulmonary hypertension. The table is illustrative, and the alternatives suggested are based on UK prescribing. Given local availability and licensing the range of options may be different in other countries.

\section{Publish your work in this journal}

The International Journal of COPD is an international, peer-reviewed journal of therapeutics and pharmacology focusing on concise rapid reporting of clinical studies and reviews in COPD. Special focus is given to the pathophysiological processes underlying the disease, intervention programs, patient focused education, and self management protocols. This journal is indexed on PubMed Central, MedLine and CAS. The manuscript management system is completely online and includes a very quick and fair peer-review system, which is all easy to use. Visit http://www.dovepress.com/testimonials.php to read real quotes from published authors. 